\title{
Nonsurgical management of ankylosing spondylitis
}

\author{
Jaypal Reddy Sangala, M.D., M.Ch., D.N.B., Elias Dakwar, M.D., Juan Uribe, M.D., \\ and Fernando Vale, M.D. \\ Department of Neurological Surgery, University of South Florida, Tampa, Florida
}

\begin{abstract}
Object. The aim of this study was to review the current evidence-based nonsurgical management strategies of ankylosing spondylitis (AS) for spine surgeons. Whereas surgical management is indicated in a highly selected group, nonsurgical management is itself a useful measure for nearly all patients with AS.

Methods. The authors conducted a literature review of PubMed using relevant search words. All the articles published in English in the last 15 years were reviewed and the level of evidence provided by them was noted.

Results. Nonpharmacological treatments in the form of physical therapy and patient education have Level Ib evidence in maintaining function in AS. There is Level Ib evidence supporting the use of nonsteroidal antiinflammatory drugs (NSAIDs) and coxibs for treatment in patients with symptoms. There is not enough evidence to support the use of conventional disease-modifying antirheumatoid arthritis drugs. Tumor necrosis factor (TNF) $\alpha$ inhibitors (infliximab, etanercept, and adalimumab) are associated with Level Ib evidence in improving spinal pain, function, inflammatory biomarkers, and spinal inflammation detected by magnetic resonance imaging in patients in whom symptom duration has exceeded 3 months.

Conclusions. Physical therapy and patient education are useful for all patients diagnosed with AS. If symptomatic, patients are started with either a course of nonselective NSAIDs or a selective cyclooxygenase-2 inhibitor. The role of NSAIDs as a disease-modifying therapy in the treatment of AS is increasingly being understood. The central role of TNF in the pathogenesis of AS is now known, and the advent of biological treatment in the form of anti-TNF $\alpha$ factors has revolutionized the medical management of AS and is used in patients with axial disease whose symptoms persist despite an adequate dose of NSAIDs. (DOI: 10.3171/FOC/2008/24/1/E5)
\end{abstract}

\section{KEY WORDS • ankylosing spondylitis • nonsurgical management • pharmacological therapy $\bullet$ tumor necrosis factor}

$\mathrm{N}$ ONSURGICAL management benefits nearly all patients with AS, and surgery is reserved for a highly selected group of patients. In the recent past, there have been promising developments in the medical management of AS. Because AS has a high prevalence in the population (range $0.1-2 \%$ ), ${ }^{5}$ affected patients can present to neurosurgeons with neurosurgical disorders unrelated to AS. To provide comprehensive care to patients with AS requiring neurosurgical care, it is important for all neurosurgeons and, more importantly, spine surgeons to be well versed in nonsurgical management of this entity. In this article we give an overview of the current evidence-based nonsurgical management of AS. Relevant pathophysiology

Abbreviations used in this paper: AS = ankylosing spondylitis; ASAS/EULAR $=$ ASsessment in Ankylosing Spondylitis/EUropean League Against Rheumatism; BASDAI = Bath Ankylosing Spondylitis Disease Activity Index; $\mathrm{COX}=$ cyclooxygenase; DMARD = disease-modifying antirheumatic drug; FDA = Food and Drug Administration; IL = interleukin; NSAID = nonsteroidal antiinflammatory drug; TNF $=$ tumor necrosis factor. of the disease is reviewed to underscore the targets of the various pharmacological agents.

\section{Pathology and Site of Action of Pharmacological Agents}

Ankylosing spondylitis is a chronic, immune-mediated inflammatory disease that is associated with inflammation in the sacroiliac joints, the axial skeleton, entheses, peripheral joints, uvea, and other structures. The initial pathological changes are initiated by macrophage infiltration that leads to synovitis and enthesitis. The macrophages are later replaced by lymphocytes, leading to release of a large number of inflammatory mediators, most significant of which are TNF, vascular endothelial growth factor, and IL-1. These inflammatory mediators lead to bone erosion, pannus, and granulation tissue formation. The eroded bone heals by osseous ankylosis. To prevent these pathological changes, a number of steps can be targeted by various pharmacological agents (Fig. 1). Briefly, the nonsurgical treatment modalities can be classified into 7 groups. ${ }^{20}$ Clinically, all of these factors work to reduce pain and/or 
Macrophages infiltration

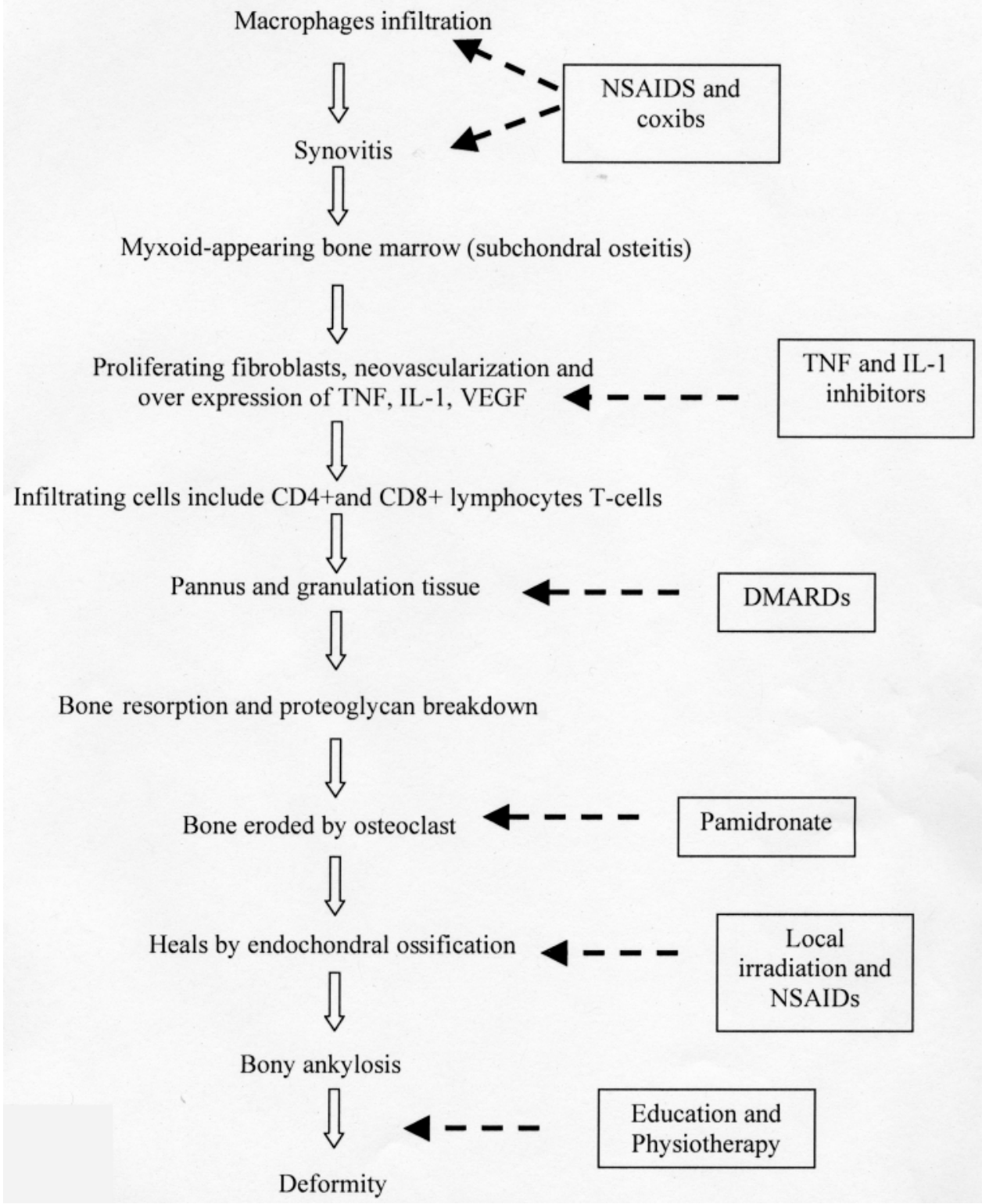

FIG. 1. Flow chart of series of pathological changes and site of action of the various treament modalities. Abbreviation: VEGF $=$ vascular endothelial growth factor.

improve function ${ }^{27}$ to a varying extent: 1) physiotherapy and education; 2) simple analgesics; 3) NSAIDs and coxibs 4) local and systemic corticosteroids; 5) radiotherapy; 6) DMARDs; 7) biological treatments (TNF and IL-1 inhibitors).

\section{Nonsurgical Management}

\section{Nonpharmacological Treatment}

Physical Therapy. Physical therapy in the form of home exercises has been shown to improve function (Level $\mathrm{Ib}$ evidence) in the short term as reported by Dagfinrud et al. ${ }^{10}$ in a Cochrane database review. However, it is still not clear which treatment protocol (group physical therapy, home exercises, or a combination) should be recommended in the management of AS. Balneotherapy (treatment by bathing) has been shown in a randomized clinical trial to have a supplementary effect on improvement in disease activity and functional parameters in patients with AS immediately after the treatment period. ${ }^{1}$ No study, however, has been conducted to determine the long-term benefits of these interventions. Although spa therapy is popular, there are no good studies supporting this intervention. ${ }^{9}$

Patient Education. At the 12-month follow-up examination Basler and Rehfisch ${ }^{2}$ found that cognitive-behavioral therapy had reduced patients' pain intensity, anxiety, and psychophysiological symptoms. Although pain reduction was statistically significant, it did not exceed $14 \%$ in the pain diary. The more important aspect of the treatment appeared to be emotional stabilization and increased feelings of well-being. Group education has also been shown to be useful. ${ }^{26}$ 
TABLE 1

Summary of ASAS/EULAR proposals for the management of AS

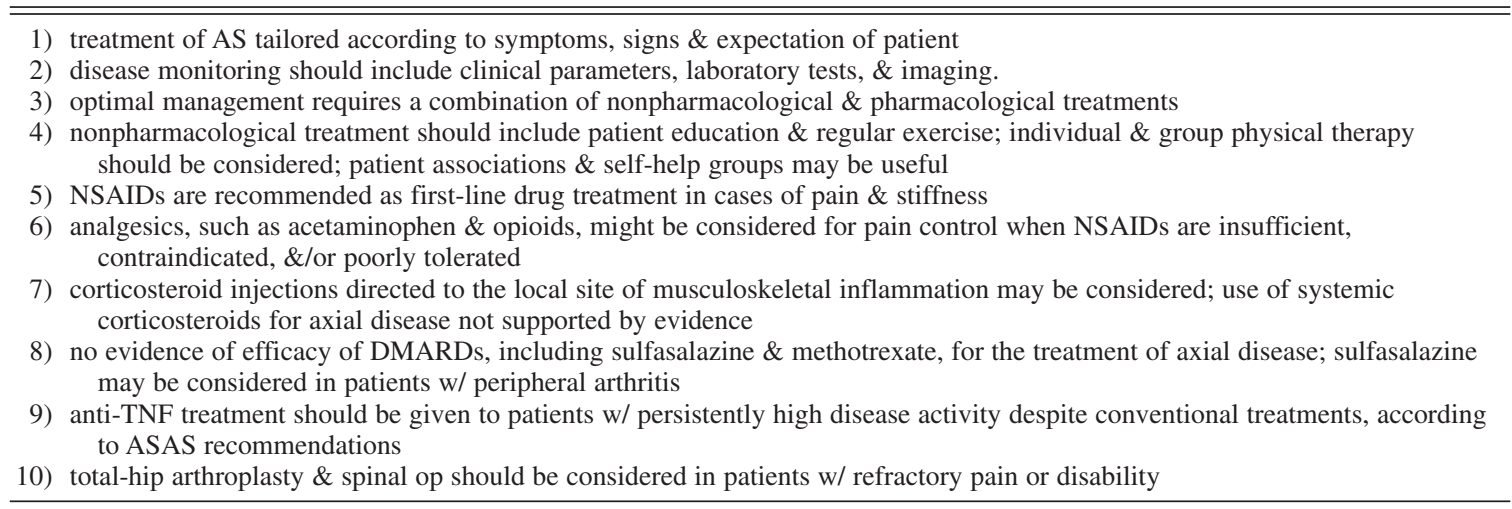

\section{Pharmacological Treatment}

Simple Analgesics and Opioids. Analgesics such as paracetamol can reduce pain and thus improve a patient's functional capacity. However, there is insufficient evidence in the literature to support the aforementioned assertion outright. The only available study was performed by $\mathrm{Pal},{ }^{17}$ in which the author found that simple analgesic agents were less effective than NSAIDs for controlling pain in AS. Opioids can be used as alternatives to NSAIDs or as supplementary analgesics. ${ }^{12}$

Nonsteroidal Antiinflammatory Drugs and Coxibs. Nonsteroidal antiinflammatory drugs continue to be the first-line treatment in AS. ${ }^{25}$ After diagnosis, symptomatic patients are started on a course of NSAID therapy, with close monitoring of the clinical response. There is Level Ib evidence that NSAIDs improve spinal pain and function in the short term. ${ }^{27}$ Although conventionally these groups of drugs have been considered symptom-modifying agents, there is now evidence that they also are disease-modifying agents. ${ }^{24}$ The inhibition of radiographic progression by continuous intake of NSAIDs can be explained by their inhibition of prostaglandins; however, this proposition warrants further study. Several in vitro studies and animal investigations have shown impaired bone healing in the presence of NSAIDs. The steps associated with bone fusion include an inflammatory response, chondrogenesis, and finally bone formation. Through inhibition of COX and subsequently prostaglandins, NSAIDs may inhibit new bone formation. ${ }^{19}$ This inhibition is clinically used to prevent postoperative ossification. There may be differences related to the degree of COX-1 and COX-2 inhibition by the various NSAIDs. Coxibs are a group of NSAIDs with selective COX-2 inhibition. An inducible enzyme, COX-2 converts arachidonic acid to prostaglandin E2, a modulator of bone metabolism. A cytoprotective enzyme, $\mathrm{COX}-1$ is present in the gastric mucosa, kidneys, and heart. Nonsteroidal antiinflammatory drugs cause increased gastrointestinal bleeding, which is dose dependent and can be reduced with gastroprotective agents such as misoprostol, double doses of H2 blocker, and proton pump inhibitors. Cardiovascular toxicity with coxibs and NSAIDs is now well established, which has led to withdrawal of certain NSAIDs from the market. ${ }^{27}$

Local and Systemic Corticosteroids. There is Level Ib evi- dence supporting the pain-improving efficacy of intra- and periarticular steroid injections into the sacroiliac joints. ${ }^{15}$ There is no evidence of any role for intraarticular steroid injections into other regions of the spine. ${ }^{27}$ There is also no evidence of any role for the systemic use of steroids in $\mathrm{AS}^{27}$

\section{Disease-Modifying Antirheumatic Drugs}

Sulfasalazine. The best investigated DMARD for the treatment of AS is sulfasalazine. Chen and $\mathrm{Liu}^{7}$ performed a metaanalysis of the role of sulfasalazine in AS and concluded that it exhibited some benefit in reducing the erythrocyte sedimentation rate and easing spinal stiffness in all patients with AS but that it showed no evidence of benefit in physical function, pain, spinal mobility, enthesitis, or patient and physician global assessment. The authors reported that there were more withdrawals for side effects (relative risk 1.47, 95\% confidence interval 1.01-2.13) in the sulfasalazine group than in the placebo group, although severe side effects were rare. They also concluded that patients in whom the disease was seen at an early stage - with higher erythrocyte sedimentation rate (or active disease) and with peripheral arthritis — might benefit from sulfasalazine treatment. There is also evidence that sulfasalazine prevents attacks of AS-associated uveitis. ${ }^{27}$ Hence, at the moment there is only limited evidence that sulfasalazine has an overall efficacy in AS, with a possible effect in early and peripheral arthritis.

Methotrexate. Methotrexate is an antimetabolite that prevents the conversion of bihydrofolic acid to tetrahydrofolic acid. Although the authors of a few small open studies involving methotrexate have reported a potential effect in $\mathrm{AS}$, a metaanalysis performed by Chen and $\mathrm{Liu}^{8}$ failed to reveal that it had any role. No placebo-controlled studies have been performed to date.

Aside from case reports there are no studies involving patients with AS receiving other DMARDs that are effective in the treatment of rheumatoid arthritis such as gold, azathioprine, cyclosporine $\mathrm{A}$, or leflunomide. ${ }^{27}$

\section{Biological Treatments}

Tumor Necrosis Factor Inhibitors. Tumor necrosis factor is a proinflammatory cytokine that has been labeled as a 
"master regulator" of the immune response. ${ }^{23}$ This cytokine is produced primarily by macrophages and activated lymphocytes; production increases in response to inflammation, infection, or injury and results in the upregulated expression of a multitude of cytokines, including IL-1 and IL-6, which stimulate bone resorption and proteoglycan breakdown. ${ }^{5}$ Elevated levels of TNF mRNA and protein are found in biopsy specimens of the sacroiliac joints obtained in patients with AS. In addition, mice that express a truncated form of TNF develop an inflammatory polyarthritis resembling AS, which can be resolved with TNFblocking therapy.

Three anti-TNF agents have been studied and 2 of these agents are approved by the FDA for the treatment of AS: etanercept, infliximab, and adalimumab. ${ }^{5}$

Infliximab. Infliximab is a monoclonal chimeric human anti-TNF antibody that binds with high affinity to TNF. It is administered by intravenous infusion of $5 \mathrm{mg} / \mathrm{kg}$ body weight at baseline ( 0 weeks), 2 weeks, and 6 weeks and then every 6 weeks. It has shown to be clinically and radiologically effective and has been approved by the FDA., ${ }^{3,22}$

Etanercept. Etanercept is a dimeric fusion protein of the TNF receptor linked to the Fc portion of human immunoglobulin G1. It is a recombinant $\mathrm{p} 75$ receptor fusion $75-\mathrm{kD}$ TNF protein that acts competitively to inhibit the cell surface receptor binding of TNF. Etanercept is administered by subcutaneous injection at a dose of $25 \mathrm{mg}$ twice weekly. It has been shown to be clinically and radiologically effective and has been approved by the FDA. ${ }^{6,11}$

Adalimumab. Adalimumab is a monoclonal, fully human anti-TNF antibody that binds with high affinity to TNF. It is approved for the treatment of active rheumatoid arthritis in the US. It is given by subcutaneous injection at a dose of $40 \mathrm{mg}$ every 2 weeks or weekly. It has been shown to be clinically and radiologically effective but has not yet been approved by the FDA. ${ }^{13}$

\section{Initiation of Anti-TNF Therapy}

The indication for the initiation of anti-TNF therapy is failure of standard treatments. ${ }^{4}, 18$ Before anti-TNF therapy can be initiated, however, disease activity should score at least 4 on the BASDAI (score range $0-10$ ), with a physician's global assessment score of at least 2 (mild disease) on the Lickert Scale. Patients must have failed to respond to treatment involving the use of 2 NSAIDs during a 3month period (due to intolerance or lack of efficacy). If patients have peripheral arthritis or enthesitis, a DMARD such as sulfasalazine or methotrexate must also have failed to resolve symptoms. If these criteria are met, patients should be treated with etanercept $(25 \mathrm{mg}$ twice weekly or $50 \mathrm{mg}$ once weekly) or infliximab $(5 \mathrm{mg} / \mathrm{kg}$ body weight, intravenously infused at baseline, 2 weeks, 6 weeks, and then every 6 weeks thereafter). Before starting anti-TNF treatment, patients must be screened for the presence of latent tuberculosis in accordance with the standard recommendations. A patient's response to treatment is evaluated at 6 weeks and 12 weeks and is defined as an improvement of at least 50\% in BASDAI score or an absolute change of $2 \mathrm{~cm}$ on a $0-10$ visual analog scale, as well as a physical global assessment change of at least 1 Lickert unit.

Effects on Disease Progression. There are convincing data to show that anti-TNF therapy significantly reduces clinically and radiologically documented disease., ${ }^{3,11,13}$ This sustained response has been observed during nearly 2 years of etanercept therapy. Improvements in magnetic resonance imaging-based scores were persistently observed at 24 weeks. In several studies TNF inhibitors have also been shown to improve bone mineral density. Not only were TNF blockers associated with improvements in both clinical and magnetic resonance imaging scores, but these agents have also shown to improve quality of life. ${ }^{5}$

Safety. Some of the most common adverse events are reactions to infusion, which can range from nausea, headaches, pruritis, and dizziness to hypotension, shortness of breath, and chest pain. This treatment has been associated with increased risk of infection, both common upper respiratory tract infections and opportunistic infections-in particular, tuberculosis. These patients also had higher rates of extrapulmonary tuberculosis and a higher mortality rate than individuals with tuberculosis who had not received anti-TNF $\alpha$.

Screening for Mycobacterium tuberculosis has been shown to decrease the incidence of tuberculous disease associated with TNF blockers and is now a standard prerequisite for anti-TNF $\alpha$ treatment. Other serious opportunistic infections in patients who received anti-TNF $\alpha$ therapy include histoplasmosis, coccidiomycosis, and blastomycosis. Demyelinating disease, lupuslike syndromes, and worsening of preexistent congestive heart failure have also been reported in case series, although precise incidences are not known. ${ }^{15}$

\section{Other Treatments of AS}

Investigators have evaluated anakinra, an IL-1 inhibitor approved for the treatment of rheumatoid arthritis, in the treatment of AS. Conflicting reports are available on the efficacy of anakinra in the management of AS. ${ }^{14,21}$ Hence, larger clinical trials are required for drawing any conclusions about its efficacy.

Recommendations of Combined ASAS/EULAR Groups. The combined ASAS/EULAR task forces have proposed 10 recommendations for the management of AS (Table 1). ${ }^{26}$ These recommendations form the cornerstone of AS management. As it is for any other disease, the treatment of AS should be tailored according to the symptoms, signs, and expectations of the patient. The disease monitoring of patients should include history, clinical features, laboratory test results, and imaging findings. Some of the scoring systems used frequently in AS include the following: ASAS core sets, BASDAI, BAS Functional Index, BAS Metrology Index, 36-Item Short Form Health Survey, and the Likert Scale. The frequency of monitoring should be tailored to the individual based on symptoms, severity, and drug therapy. A combination of nonpharmacological and pharmacological treatments, including education and physical therapy, has been shown to produce the best clinical outcome. Tumor necrosis factor inhibitor therapy should be initiated according to ASAS recommendations. Hip replacement and spinal surgery have to be considered in patients with refractory pain and/or disability.

Socioeconomics/Cost Effectiveness. Despite the high costs, the clinical benefits and improvements in quality of life in 
patients with AS receiving infliximab could result in lower disease-associated costs than does standard care, which translates to a short-term cost of $\sim \$ 70,000$ US (per quality-adjusted life year gained). ${ }^{5}$ Furthermore, the daily productivity of patients with active disease, which was substantially associated with functional impairment and disease activity, could greatly improve with infliximab, and this was associated with reduced workday loss in employed patients. In a metaanalysis of the cost effectiveness of antiTNF $\alpha$ therapy, however, McLeod et al. ${ }^{16}$ found that none of the 3 agents was cost-effective in the short term. Thus, larger clinical trials are required to prove convincingly the cost effectiveness of anti-TNF $\alpha$ therapy.

\section{Conclusions}

Physical therapy and education are useful for all patients diagnosed with AS. If symptomatic, patients receive either a nonselective NSAID or a selective COX-2 inhibitor. The role of NSAIDs as disease-modifying agents in the treatment of AS is being better understood. The central role of TNF in the pathogenesis of AS is now known, and the advent of biological treatment in the form of anti-TNF factors has revolutionized the medical management of AS. These agents are used in patients with axial disease who remain symptomatic despite receiving adequate doses of NSAIDs

\section{References}

1. Altan L, Bingöl U, Aslan M, Yurtkuran M: The effect of balneotherapy on patients with ankylosing spondylitis. Scand J Rheumatol 35:283-289, 2006

2. Basler HD, Rehfisch HP: Cognitive-behavioral therapy in patients with ankylosing spondylitis in a German self-help organization. J Psychosom Res 35:345-354, 1991

3. Braun J, Baraliakos X, Brandt J, Listing J, Zink A, Alten R, et al: Persistent clinical response to the anti-TNF-alpha antibody infliximab in patients with ankylosing spondylitis over 3 years. Rheumatology (Oxford) 44:670-676, 2005

4. Braun J, Pham T, Sieper J, Davis J, van der Linden S, Dougados $\mathrm{M}$, et al: International ASAS consensus statement for the use of anti-tumor necrosis factor agents in patients with ankylosing spondylitis. Ann Rheum Dis 62:817-824, 2003

5. Braun J, Sieper J: Ankylosing spondylitis. Lancet 369: 1379-1390, 2007

6. Calin A, Dijkmans BA, Emery P, Hakala M, Kalden J, LeirisaloRepo M, et al: Outcomes of a multicentre randomised clinical trial of etanercept to treat ankylosing spondylitis. Ann Rheum Dis 63: 1594-1600, 2004

7. Chen J, Liu C: Is sulfasalazine effective in ankylosing spondylitis? A systematic review of randomized controlled trials. J Rheumatol 33:722-731, 2006

8. Chen J, Liu C: Methotrexate for ankylosing spondylitis. Cochrane Database Syst Rev 4: CD004524, 2004

9. Claudepierre P: Spa therapy for ankylosing spondylitis: still useful? Joint Bone Spine 72:283-285, 2005

10. Dagfinrud H, Kvien TK, Hagen KB: Physiotherapy interventions for ankylosing spondylitis. Cochrane Database Syst Rev 4: CD002822, 2004

11. Davis JC Jr, Van Der Heijde D, Braun J, Dougados M, Cush J, Clegg DO, et al: Recombinant human tumor necrosis factor receptor (etanercept) for treating ankylosing spondylitis: a randomized, controlled trial. Arthritis Rheum 48:3230-3236, 2003
12. Dougados M, Dijkmans B, Khan M, Maksymowych W, van der Linden S, Brandt J: Conventional treatments for ankylosing spondylitis. Ann Rheum Dis 61 (3 Suppl):40-50, 2002

13. Haibel H, Rudwaleit M, Brandt HC, Grozdanovic Z, Listing J, Kupper $\mathrm{H}$, et al: Adalimumab reduces spinal symptoms in active ankylosing spondylitis: clinical and magnetic resonance imaging results of a fifty-two-week open-label trial. Arthritis Rheum 54: 678-681, 2006

14. Haibel H, Rudwaleit M, Listing J, Sieper J: Open label trial of anakinra in active ankylosing spondylitis over 24 weeks. Ann Rheum Dis 64:296-298, 2005

15. Maugars Y, Mathis C, Berthelot JM, Charlier C, Prost A: Assessment of the efficacy of sacroiliac corticosteroid injections in spondylarthropathies: a double-blind study. Br J Rheumatol 35: 767-770, 1996

16. McLeod C, Bagust A, Boland A, Dagenais P, Dickson R, Dundar $\mathrm{Y}$, et al: Adalimumab, etanercept and infliximab for the treatment of ankylosing spondylitis: a systematic review and economic evaluation. Health Technol Assess 11:1-158, 2007

17. Pal B: Use of simple analgesics in the treatment of ankylosing spondylitis. Br J Rheumatol 26:207-209, 1987

18. Pham T, van der Heijde D, Calin A, Khan MA, van der Linden S, Bellamy N, et al: Initiation of biological agents in patients with ankylosing spondylitis: results of a Delphi study by the ASAS Group. Ann Rheum Dis 62:812-816, 2003

19. Radi ZA, Khan NK: Effects of cyclooxygenase inhibition on bone, tendon, and ligament healing. Inflamm Res 54:358-366, 2005

20. Rehart S, Kerschbaumer F, Braun J, Sieper J: [Modern treatment of ankylosing spondylitis.] Orthopade 36:963-974, 2007 (Ger)

21. Tan AL, Marzo-Ortega H, O'Connor P, Fraser A, Emery P, McGonagle D: Efficacy of anakinra in active ankylosing spondylitis: a clinical and magnetic resonance imaging study. Ann Rheum Dis 63:1041-1045, 2004

22. van der Heijde D, Dijkmans B, Geusens P, Sieper J, DeWoody K, Williamson P, et al: Efficacy and safety of infliximab in patients with ankylosing spondylitis: results of a randomized, placebocontrolled trial (ASSERT). Arthritis Rheum 52:582-591, 2005

23. Visvanathan S, Wagner CL, Marini JC, van der Heijde D, Baker $\mathrm{D}$, Gathany $\mathrm{T}$, et al: Inflammatory biomarkers, disease activity, and spinal disease measures in patients with ankylosing spondylitis after treatment with infliximab. Ann Rheum Dis [epub ahead of print], 2007

24. Wanders A, Heijde D, Landewé R, Béhier JM, Calin A, Olivieri I, et al: Nonsteroidal antiinflammatory drugs reduce radiographic progression in patients with ankylosing spondylitis: a randomized clinical trial. Arthritis Rheum 52:1756-1765, 2005

25. Zochling J, Braun J: Management and treatment of ankylosing spondylitis. Curr Opin Rheumatol 17:418-425, 2005

26. Zochling J, van der Heijde D, Burgos-Vargas R, Collantes E, Davis JC Jr, Dijkmans B, et al: ASAS/EULAR recommendations for the management of ankylosing spondylitis. Ann Rheum Dis 65:442-452, 2006

27. Zochling J, van der Heijde D, Dougados M, Braun J: Current evidence for the management of ankylosing spondylitis: a systematic literature review for the ASAS/EULAR management recommendations in ankylosing spondylitis. Ann Rheum Dis 65:423-432, 2006

Manuscript submitted October 15, 2007.

Accepted November 8, 2007.

Address correspondence to: Fernando Vale, M.D., Department of Neurological Surgery, Seventh Floor, 2A Columbia Drive, University of South Florida, Tampa, Florida 33606. email: fvale@health.usf.edu. 